\title{
Comparison of Videofluoroscopic Swallowing Study and Fiberoptic-Endoscopic Evaluation of Swallowing Findings in Pediatric Patients
}

\author{
Volkan Güngör ${ }^{1}$, Mehmet Umut Akyol ${ }^{2}$, Numan Demir ${ }^{3}$, Nilda Süslü², Selen Serel Arslan ${ }^{3}$ \\ ${ }^{1}$ Department of Otorhinolaryngology Head and Neck Surgery, Ordu University Education and Research Hospital, Ordu, Turkey \\ ${ }^{2}$ Department of Otorhinolaryngology Head and Neck Surgery, Hacettepe University Faculty of Medicine, Ankara, Turkey \\ ${ }^{3}$ Department of Physiotherapy and Rehabilitation, Hacettepe University Faculty of Health Science Ankara, Turkey
}

Received: 11 November 2020, Accepted: 09 December 2020, Published online: 31 December 2020

(C) Ordu University Institute of Health Sciences, Turkey, 2020

\begin{abstract}
Objective: This study is designed to determine the value and accuracy of Fiberoptic Endoscopic Evaluation of Swallowing (FEES) in the diagnosis of swallowing disorders as a diagnostic tool, in comparison with the widely accepted Videofluoroscopic Swallowing Study (VFSS) in pediatric patients.

Methods: Cross-sectional study in tertiary referral center. Fifty one children with swallowing difficulty due to various diseases were prospectively evaluated using both VFSS and FEES. The variables, early pharyngeal spillover, pharyngeal residues, laryngeal sensitivity-silent aspiration, laryngeal penetration and laryngeal aspiration were evaluated in all patients.

Results: Mean patient age was $29.8 \pm 17.8$ (range 9-72) months. Six patients were younger than 13 months, 19 were between 13 and 24 months, and 26 patients were older than 2 years of age. There were 21 (42\%) females and $30(58 \%)$ males in the study group. Significant correlation in pharyngeal residues and laryngeal sensitivity-silent aspiration findings were found between FEES and VFSS data. No Significant dissimilarity in laryngeal aspiration and penetration evaluation was found.

Conclusion: Swallowing evaluation in children is more challenging than adults. Study findings showed that FEES outcomes correlate with VFSS data, especially in the diagnosis of laryngeal aspiration and FESS is a valuable tool in identifying swallowing disorders in pediatric patients.

Key words: Swallowing disorders, Fiberoptic endoscopic evaluation of swallowing, Dysphagia, Videofluoroscopic Swallowing Study, Laryngeal aspiration.

Suggested Citation: Gungor V, Akyol MU, Demir N, Suslu N, Arslan SS. Comparison of Videofluoroscopic Swallowing Study and Fiberoptic-Endoscopic Evaluation of Swallowing Findings in Pediatric Patients. Middle Black Sea Journal of Health Science, 2020; 6(3):390-396
\end{abstract}

Address for correspondence/reprints:

DOI: $\quad 10.19127 / \mathrm{mbsjohs} .822913$

Volkan Güngör

Telephone number: +90 (533) 5259300

ORCID-ID 0000-0003-1237-9751

E-mail: vgungor@outlook.com 


\section{Introduction}

Genetic malformations, neurological and systemic diseases are frequently associated with pathologic feeding and swallowing in children. In these patient's gastro-esophageal reflux and aspiration pneumonia due to swallowing disorders can be seen frequently, causing significant mortality and morbidity (Darrow and Harley 1998).

Videofluoroscopic swallowing study (VFSS) which has long been viewed as the "gold standard"(Logeman 1983.; Langmore et al. 1988; Langmore 2017; Re et al. 2019) and fiberoptic endoscopic evaluation of swallowing (FEES) are the most commonly used methods for the diagnosis and follow-up of swallowing disorders. Both of these instrumental studies are utilized to determine the deficit in swallowing and feeding, causing airway contamination. Ability to observe all swallowing phases, including the oral preparatory, oral transit times, upper esophageal sphincter opening and esophageal transit time and assessing the position of hyoid-larynx complex are major superiorities of VFSS. On the other hand, radiation exposure is the main limitation of VFSS (Jones 1999; Bluestone 2003; Ko et al. 2019), especially in the pediatric population. Patients who have chronic diseases such as hypoxic ischemic encephalopathy may require multiple radiologic examinations which increase the cumulative radiation exposure. FEES have some advantages such as no radiation exposure, less cost, the opportunity of bedside evaluation, diagnostic power for anatomical features of the larynx and is performed routinely in the pediatric population by otolaryngologists to diagnose various concomitant pathologies. Briefly, these techniques cannot provide identical diagnostic information across all aspects of swallowing and both methods are known to be useful and complementary to each other. In adults, both methods have been shown to be equally effective by many studies (Kidder et al. 1994; Langmore et al.1988; Kaye et al. 1997; Wu et al. 1997; Colodny 2002; Giraldo-Cadavid et al. 2017). However, in the English language literature there are few studies including infants and children (Leder and Karas 2000; da Silva et al. 2010; Reynolds et al. 2016; Miller and Willging 2020) that compare the accuracy and reliability of these methods.

The aim of the present study is to investigate the place and accuracy of FEES in swallowing disorders in children by comparing FEES findings with the widely accepted VFSS in a pediatric population with swallowing dysfunction.

\section{Methods}

\section{Subjects and Study Design}

This study was approved by the Committee of Ethics of Hacettepe University, School of Medicine (Approval number: HEK 11/32-3). Children and guardians of patients were informed about the study and a signed consent form.

Fifty-one children referred to our clinic with dysfunctional swallowing or associated respiratory problems mainly from pediatric gastroenterology, pediatric neurology and pediatric pulmonology departments were prospectively evaluated using VFSS and FEES between April 2011 and November 2011 at Hacettepe University, School of Medicine, Otorhinolaryngology and Radiology departments. Diagnostic value of FEES was evaluated in comparison with VFSS as the gold standard method for dysphagia assessment.

\section{Procedures and equipment}

The basic FEES protocol was performed at the outpatient department, with the patient in sitting position either alone or held by one of the parents. All food used during the tests was dyed with methylene blue for better visualization. Approximately $5-\mathrm{mL}$ boluses of food with purée consistency (yogurt with methylene blue) and 5-mL boluses of food with liquid consistency (milk or water with methylene blue) were given during FEES procedures. All FEES procedures were carried out by an experienced pediatric otolaryngologist and were videotaped. FEES was performed with a Storz $3.2 \mathrm{~mm}$ nasopharyngolaryngoscope and Storz halogen light source (Karl Storz GmbH \&Co., Germany). The captured images were transferred to a Sony video monitor and recorded onto a Sony videocassette (Sony Corp., Tokyo, Japan).

Videofluoroscopic swallowing evaluation was performed with food samples of the same quantity and consistency (puree and liquid). During the radiographic study, patients were seated and viewed in the lateral projection. The fluoroscopic tube was focused on the lips anteriorly, the cervical vertebrae posteriorly, the soft palate superiorly, and the cervical esophagus inferiorly (J.A. Pro-ed,1998: 169-185.)

The videofluoroscopic studies were recorded on a DVD. 


\section{Compared Swallowing Parameters}

The following nine parameters were compared:

1- pharyngeal spillover,

2a- pharyngeal residue in the valleculae (purée),

$2 b$ - pharyngeal residue in the valleculae (liquid)

3a- pharyngeal residue in the pyriform sinuses (purée)

3b- pharyngeal residue in the pyriform sinuses (liquid)

4- velopharyngeal patency,

5- laryngeal sensitivity-silent aspiration,

6- laryngeal penetration (food or contrast residues above the vocal folds),

7- laryngeal aspiration (food or contrast residues below the vocal folds).

Laryngeal reflex assessment in FEES was used as an analogue to silent aspiration in VFSS. After larynx visualization was obtained with the endoscope, the tip of endoscope was brought in contact with either the epiglottis or arytenoids of the patient and response of the patient in terms of vocal cord adduction was observed and recorded. If there was an absence of response, the patient was noted to have no laryngeal reflex (who was considered a candidate for silent aspiration).

\section{Statistical analysis}

Analysis of all data was performed in PASW 18.0 (IBM Inc.) statistical package program McNemar test and Receiver Operating Characteristic (ROC) Curve was used for the assessment of statistical differences between FEES and VFSS. P values under 0.05 were considered statistically significant.

\section{Results}

Mean patient age was $29.8 \pm 17.8$ (range 9-72) months. Six patients were younger than 13 months, 19 were between 13 and 24 months, and 26 patients were older than 2 years of age. There were $21(42 \%)$ females and $30(58 \%)$ males in the study group. The major cause for ENT referral was respiratory problems in 17 (\%33), gastroesophageal reflux in 5 (\%9.8), persistent vomiting in 3 (\%5.8), genetic malformations in $11(\% 21)$ and other causes (\%9.8).

All previously explained parameters of swallowing dysfunction were compared for diagnostic agreement. If non-parametric testing indicated agreement between FEES and VFSS ( $p>0.05$ ), ROC curves were also evaluated to further establish correlation for the first seven parameters. Diagnostic agreement was found between FEES and VFSS for pharyngeal spillover (1), pharyngeal residue in the valleculae (purée food) (2a), pharyngeal residue in the valleculae (liquid) (2b), pharyngeal residue in the pyriform sinuses (purée food) (3a), pharyngeal residue in the pyriform sinuses (liquid) (3b), laryngeal sensitivity-silent aspiration (5). No correlation was present regarding velopharyngeal patency (4) between the two methods. A concise summary of results regarding the first seven parameters is provided in table 1.

Laryngeal penetration and aspiration was evaluated for both purée food and liquids. While in $29(57 \%)$ patients both FEES and VFSS indicated aspiration for liquid consistency, FEES could not detect 2 instances of penetration (4\%) and 1 instance of aspiration (2\%) that was detected by VFSS. Five patients $(10 \%)$ had penetration according to FEES while according to VFSS these patients did not have penetration for liquid consistency. For purée food in 22 patients (42\%) FEES and VFSS agreed on aspiration. FEES could not detect 4 cases of penetration (\%8) and 2 cases of aspiration (4\%) which were detected by VFSS. Three patients $(6 \%)$ had penetration according to FEES while according to VFSS these patients were found normal. Crossdistribution tables for VFSS and FEES results regarding purée and liquid food consistencies can be seen in Table 2 and 3.

Sensitivity for detecting aspiration was $96 \%$ for liquids and $73 \%$ for purée food. Specificity for detecting aspiration was $95 \%$ for liquids and $85 \%$ for puree food. Sensitivity for detecting aspiration or penetration was $90 \%$ for liquid consistency and $81 \%$ for puree consistency. Specificity for detecting aspiration or penetration was $72 \%$ for liquid consistency and $84 \%$ for puree consistency. Sensitivity and specificity values for FEES can be seen in Table 4. 
Table 1. Statistical analyses for early pharyngeal spillover, Residues for vallecula and pyriform sinuses, velopharyngeal patency and laryngeal sensitivity-silent aspiration

\begin{tabular}{lcc}
\hline Compared parameter & $\mathbf{p}^{*}$ values & Area Under Curve $^{* * *}$ \\
\hline 1-Early pharyngeal spillover & 0.453 & 0.783 \\
\hline 2a-Residues for vallecula (purée food) & 0.990 & 0.887 \\
\hline 2b-Residues for vallecula (liquid) & 0.996 & 0.769 \\
\hline 3a-Residues for pyriform sinuses (purée food) & 0.219 & 0.863 \\
\hline 3b-Residues for pyriform sinuses (liquid) & 0.998 & 0.880 \\
\hline 4-Velopharyngeal patency & 0.012 & 0.785 \\
\hline 5-Laryngeal sensitivity (FEES)-Silent aspiration (VFSS) & 0.754 & \\
\hline
\end{tabular}

${ }^{*}$ McNemar Test ${ }^{* *}$ ROC curve

Table 2. Aspiration-Penetration cross- tabs for liquid consistency

\begin{tabular}{llcccc}
\hline & & \multicolumn{3}{c}{ VFSS } & Total \\
\cline { 2 - 5 } FEES & Normal & Penetration & Aspiration & \\
\cline { 2 - 5 } & Normal & $13(\% 25)$ & $2(\% 4)$ & $1(\% 2)$ & 16 \\
\cline { 2 - 5 } & Penetration & $5(\% 10)$ & 0 & 0 & 5 \\
\hline Aspiration & 0 & $1(\% 2)$ & $29(\% 57)$ & 30 \\
\hline Total & & 18 & 3 & 30 & 51 \\
\hline
\end{tabular}

(McNemar Test, $\mathrm{P}=0.350>0.05$ )

Table 3. Aspiration-Penetration cross- tabs for puree consistency.

\begin{tabular}{llcccc}
\hline & & \multicolumn{3}{c}{ VFSS } & Total \\
\cline { 3 - 5 } FEES & Normal & Penetration & Aspiration & \\
\cline { 2 - 5 } & Normal & $16(\% 32)$ & $2(\% 4)$ & $4(\% 8)$ & 22 \\
\cline { 2 - 6 } & Penetration & $3(\% 6)$ & 0 & $2(\% 4)$ & 5 \\
\hline Aspiration & 0 & $2(\% 4)$ & $22(\% 42)$ & 24 \\
\hline
\end{tabular}

(McNemar Test, $\mathrm{p}=0.241>0.05)$

Table 4. Sensitivity and specificity values for FEES

\begin{tabular}{|c|c|c|}
\hline Paramaters & Sensitivity (\%) & Specificity (\%) \\
\hline \multicolumn{3}{|l|}{ Detection of aspiration } \\
\hline Liquid & $96 \%$ & $95 \%$ \\
\hline Puree & $73 \%$ & $85 \%$ \\
\hline \multicolumn{3}{|l|}{$\begin{array}{l}\text { Detection of aspiration or } \\
\text { penetration }\end{array}$} \\
\hline Liquid & $90 \%$ & $72 \%$ \\
\hline Puree & $81 \%$ & $84 \%$ \\
\hline
\end{tabular}

\section{Discussion}

While the use of FEES is relatively well known for swallowing evaluation, there is a need to show the accuracy of this procedure in the pediatric patient population. In this study, we aimed to determine the reliability of FEES in the pediatric population by correlating this method with VFSS which is considered a gold standard evaluation for swallowing disorders. We found good correlation between FEES and VFSS to detect early pharyngeal spillover, residue in valleculae and pyriform sinuses, and laryngeal sensitivity-silent aspiration. Penetration and aspiration were also reliably determined with both methods.

There has been an interest in endoscopic evaluation of swallowing since Langmore et al. 
(1983) described FEES in 1983. In 1991 Langmore et al (1991) published the first study comparing FEES and VFSS as the gold standard in adults and showed high levels of agreement in diagnosis for adult patients. Likewise, in children, VFSS is still regarded as the standard diagnostic method by several authors (Arvedson and Lefton-Greif 2007; Kramer and Eicher 1993; Newman et al. 1991). But in spite of the variety of studies on this subject conducted for adults (Kaye et al.1997; J.A. Pro-ed,1998: 169-185.) which compare FEES with VFSS, in the current literature there are only two prospective studies which evaluate the value of FEES in swallowing disorders in children (Leder and Karas 2000; da Silva et al. 2010). One of the mentioned studies includes only seven patients and evaluates only laryngeal aspiration and penetration. Sensitivity and specificity values seem different between two studies. Contradictory findings from these studies prompted both authors to recommend further investigation with larger sample sizes.

Leder and Karas (Langmore et al.1991) showed the value of FEES as a diagnostic tool beside VFSS in their study for the first time in 2000. In their study, 7 patients were tested with both VFSS and FEES and there was $100 \%$ agreement in detecting laryngeal penetration and aspiration between the two methods. No other parameters were compared in this study.

In 2010, Andréa P. da Silva at al. (2010) evaluated the diagnostic value of FEES performed by two independent observers against VFSS in 30 patients. In this study FEES yielded good interobserver agreement for all parameters which demonstrates that FEES is a method with reproducible results. On the other hand, consistency between FEES and VFSS results for both of the two observers have been found to be low, indicating less than ideal diagnostic agreement between the two methods. Detecting laryngeal penetration and aspiration yielded the highest specificity and positive predictive value when compared to VFSS. The pharyngeal residue parameters and early spillover parameters show moderate correlation in this study for the worse of the observers testing with liquids. For the better observer pharyngeal residue (Sensitivity: $83 \%$ and Specificity: $79 \%$ ) and early spillover (Sensitivity: $44 \%$ and Specificity: 76\%) shows better correlation for liquids. Similarly, in our study, early pharyngeal spillover demonstrated good correlation between the two methods according to ROC analyses (AUC: 0.783). Compared to this study, pharyngeal residues for both liquid and purée food also show better agreement.

Our specificity and sensitivity values for laryngeal aspiration and penetration seem similar with Leder and Karas's findings. While da Silva et al. shows better specificity (100\%) for laryngeal aspiration with purée food than our study (\%85), our results (Sensitivity:96\% and Specificity:95\%) are better than this study (Sensitivity: 27\% and Specificity: $91 \%$ ) for liquids. In addition, Langmore et al. (1991) also reported good results (Sensitivity:88\% and Specificity:92\%) for laryngeal aspiration in adults. Despite these optimistic results for detection of aspiration, we have six patients for purée food (12\%) and 3 patients for liquids $(6 \%)$ that were considered normal according to FEES while having aspiration or penetration in VFSS. In the pediatric population, 12\% undetected aspiration is quite significant and larger samples are needed to discuss the weaknesses of FEES that might lead to misdiagnosis.

Laryngeal sensitivity can also be assessed by endoscopic examination; this is accomplished by directly stimulating various laryngeal areas with the tip of the endoscope. This evaluation can also be accomplished by the rhythmic administration of air in a sequence of pressures, to elicit laryngeal adduction and consequently establish the sensitivity threshold (Willging and Thompson 2005; Nacci et al. 2008). In our study we evaluate laryngeal sensitivity by direct stimulation of laryngeal mucosa by contact with the tip of the endoscope and we have considered absent response to stimulation as impaired laryngeal sensitivity. We observed impaired laryngeal sensitivity outcomes of FEES correlates well with silent aspiration in VFSS. Leder at al. (1998) used FEES for diagnosis of silent aspiration in their study. In our study we demonstrate the relationship between FEES findings of laryngeal sensitivity and silent aspiration of VFSS in pediatric population. This may suggest that silent aspiration may be predicted by laryngeal sensitivity testing in FEES and then patients can be referred to further evaluation by VFSS.

Transnasal flexible fiberoptic laryngoscopy is performed routinely in children and infants but using it for FEES is not common. Some of the limitations of VFSS, such as the need for cooperation of the patient during examination is also valid for FEES in the pediatric population. Additional care should be taken to avoid any airway problems for children with severe aspiration risk. Our study showed that FEES can be used in children to avoid the aforementioned limitations of VFSS as a relatively safe and easy procedure without major difficulties or complications. Using FEES will give general and pediatric otolaryngologists a more active role in dysphasia diagnosis and management. 


\section{Conclusion}

Swallowing evaluation in children is more challenging than adults. The value of FEES, a wellaccepted diagnostic test, has only been investigated twice in the literature. Results of these studies could not agree on the level of diagnostic reliability of FEES in children. In our study, good agreement levels between FEES and VFSS support the use of FEES as a valuable diagnostic method in pediatric patients with swallowing disorders.

Ethics Committee Approval: This study was approved by the Committee of Ethics of Hacettepe University, School of Medicine (Approval number: HEK 11/32-3).

Peer-review: Externally peer-reviewed.

Author Contributions: Concept- V.G., N.S.S, M.U.A; Design V.G., N.D; Materials- V.G., N.D, S.S; Data Collection and Processing- V.G.; Literature Review- V. G.; Writing- V.G., N.D. S.S.; Critical Review- N.S.S, M.U.A.

Conflict of Interest: No conflict of interest was declared by the author.

Financial Disclosure: The author declared that this study hasn't received no financial support.

Note: This study has been prepared as a specialist thesis.

\section{References}

Arvedson JC, Lefton-Greif MA. 'Ethical and legal challenges in feeding and swallowing intervention for infants and children', Seminars in speech and language, 2007;28: 232-8.

Colodny N. 'Interjudge and intrajudge reliabilities in fiberoptic endoscopic evaluation of swallowing (Fees ${ }^{\circledR}$ ) using the Penetration-Aspiration Scale: a replication study', Dysphagia, 2002;17: 308-15.

da Silva AP et al. Comparison between videofluoroscopy and endoscopic evaluation of swallowing for the diagnosis of dysphagia in children. Otolaryngology-Head and Neck Surgery, 2010;143.2: 204-209.

Darrow DH, and Harley CM. 'Evaluation of swallowing disorders in children', Otolaryngologic clinics of North America, 1998; 31: 405-18.

Giraldo-Cadavid LF, Leal-Leano LR., Leon-Basantes GA, Bastidas AR, Garcia R, Ovalle S, and Abondano-Garavito JE. 'Accuracy of endoscopic and videofluoroscopic evaluations of swallowing for oropharyngeal dysphagia', The Laryngoscope, 2017; 127: 2002-10.
Jones B. 'Radiologic evaluation of the dysphagic patient', Nutrition in clinical practice, 1999. ;14: S10-S12.

Kaye GM, Richard DZ, and Soly B. 'Role of flexible laryngoscopy in evaluating aspiration', Annals of Otology, Rhinology \& Laryngology, 1997; 106: 705-09.

Kidder TM, Langmore SE, and Martin BJ. 1994. 'Indications and techniques of endoscopy in evaluation of cervical dysphagia: comparison with radiographic techniques', Dysphagia, 1994; 9: 256-61.

Ko EJ, Sung IY, Choi KH, Kwon YG, Yoon J, and Kim T. 2019. 'Radiation exposure during videofluoroscopic swallowing studies in young children', Int J Pediatr Otorhinolaryngol, 2019;121: 1-5.

Kramer SS, and Eicher PM. 1993. 'The evaluation of pediatric feeding abnormalities', Dysphagia, 1993; 8: $215-24$

Langmore SE , 'History of Fiberoptic Endoscopic Evaluation of Swallowing for Evaluation and Management of Pharyngeal Dysphagia: Changes over the Years', Dysphagia, 2017; 32: 27-38.

Langmore SE, Schatz K, and Olsen N.. 'Fiberoptic endoscopic examination of swallowing safety: a new procedure', Dysphagia, 1988; 2: 216-9.

Langmore SE, Schatz K, and. Olson N 1991. 'Endoscopic and videofluoroscopic evaluations of swallowing and aspiration', The Annals of otology, rhinology, and laryngology, 1991; 100: 678-81.

Langmore SE, Schatz MAK, and Nels O. 1988. 'Fiberoptic endoscopic examination of swallowing safety: a new procedure', Dysphagia, 1988; 2: 21619.

Leder SB., and Karas DE. 'Fiberoptic endoscopic evaluation of swallowing in the pediatric population', The Laryngoscope, 2000;110: 11326.

Leder SB, Sasaki CT, and Burrell MI. 'Fiberoptic endoscopic evaluation of dysphagia to identify silent aspiration', Dysphagia, 1998; 13: 19-21.

Logeman JA.. 'Evaluation and Treatment of Swallowing Disorders.', San Diego, CA: CollegeHill Press;. 1983

Logemann JA. Evaluation and treatment of swallowing disorders (Austin,Texas). Proed, 1998; 169-185.

Miller CK. and Willging JP. 'Fiberoptic Endoscopic Evaluation of Swallowing in Infants and Children: Protocol, Safety, and Clinical Efficacy: 25 Years of Experience', The Annals of otology, rhinology, and laryngology, 2020; 129: 469-81. 
Nacci A Et al. 2008. 'Fiberoptic endoscopic evaluation of swallowing (FEES): proposal for informed consent', Acta otorhinolaryngologica Italica: organo ufficiale della Societa italiana di otorinolaringologia e chirurgia cervico-facciale, 2008; 28: 206-11.

Newman LA., Cleveland RH, Blickman JG, Hillman RE, and Jaramillo D. 'Videofluoroscopic analysis of the infant swallow', Investigative radiology, 1991; 26: 870-3.

Re GL. et al. 'Swallowing evaluation with videofluoroscopy in the paediatric population', Acta otorhinolaryngologica Italica: organo ufficiale della Societa italiana di otorinolaringologia e chirurgia cervico-facciale, 2019; 39: 279-88.

Reynolds J, Carroll S, and Sturdivant C. 'Fiberoptic Endoscopic Evaluation of Swallowing: A Multidisciplinary Alternative for Assessment of Infants with Dysphagia in the Neonatal Intensive Care Unit', Adv Neonatal Care, 2016; 16: 37-43.

Tanaka N, Ueda K, Katayama A, Ushio T, Fujii M, Sakai T. 'Effect of aspiration on the lungs in children: a comparison using chest computed tomography findings. ' BMC pediatrics, 2019; 19(1), 162.

Willging JP, and Thompson DM. 'Pediatric FEESST: fiberoptic endoscopic evaluation of swallowing with sensory testing', Current gastroenterology reports, 2005; 7: 240-3.

Wu CH, Hsiao TY, Chen JC, Chang YC, and Lee SY. 1997. 'Evaluation of swallowing safety with fiberoptic endoscope: comparison with videofluoroscopic technique', The Laryngoscope, 1997; 107: 396-401. 